International Game Theory Review

(c) World Scientific Publishing Company

\title{
Indices of criticality in simple games
}

\author{
Marco DALL'AGLIO \\ LUISS University, Department of Economics and Finance, Viale Romania 32, 00197 Roma, \\ Italy-mdallaglio@luiss.it \\ Vito FRAGNELLI \\ University of Eastern Piedmont, Department of Sciences and Innovative Technologies (DISIT), \\ Viale T. Michel 11, 15121 Alessandria, Italy - vito.fragnelli@uniupo.it \\ Stefano MORETTI \\ Université Paris Dauphine, PSL Research University, CNRS, LAMSADE, 75775 Paris, France \\ - stefano.moretti@dauphine.fr \\ Received (Day Month Year) \\ Revised (Day Month Year)
}

\begin{abstract}
We generalize the notion of power index for simple games to different orders of criticality, where the order of criticality represents the possibility for players to gain more power over the members of a coalition thanks to the collusion with other players. We study the behaviour of these criticality indices to compare the power of different players within a single voting situation, and that of the same player with varying weight across different voting situations. In both cases we establish monotonicity results in line with those of Turnovec (1998). Finally, we examine which properties characterizing the indices of Shapley-Shubik and Banzhaf are shared by these new indices.
\end{abstract}

Keywords: simple games; power indices; criticality; weighted majority games; monotonicity.

Subject Classification: 91A12, 91A80.

\section{Introduction}

In the theory of simple games, power indices based on marginal contributions Straffin [1977]; Dubey et al. [2081] are used to convert information about winning and losing subsets (i.e., coalitions) of the player set into a personal attribution (of power) to each of the players, keeping into account the criticality of players over all possible coalitions (a player $i$ is considered critical for a coalition $S$ containing $i$, if $S$ is winning and $S$ without $i$ is not). However, the notion of criticality itself is not sufficiently rich to encompass all the interaction possibilities among players and, consequently, the indices based on this notion are not able to distinguish the role of players across quite different simple games. Consider for instance two simple games with only two players $i$ and $j$, and such that in the first game only the grand coali- 
tion $\{i, j\}$ is winning, whereas in the second game all coalitions, except the empty set, are winning. The Shapley-Shubik power index Shapley [1953] (or the Banzhaf one Banzhaf [1965]) yield the same power to both players and in both games, and this may be basically seen as the consequence of the fact that each player is critical only once in both situations (precisely, each player is only critical for coalition $\{i, j\}$ in the first game, and only for the corresponding singleton coalition in the second one; see Example 1 for more details). On the other hand, without any further assumption about the probability to form coalitions of different size, the two situations are quite different for the following reason: Suppose that the grand coalition $\{i, j\}$ forms; in the first game, the grand coalition may be threatened by both players, while in the second game, this is not true anymore, and each player becomes critical only if the other leaves the grand coalition. Of course, an answer to the question whether a player is more powerful in one game than in the other one is highly context-specific. Nevertheless, we can argue that the information contained in the characteristic function of a simple game cannot be adequately represented by a single attribute which exclusively relies on the notion of criticality.

In order to introduce a more general concept of criticality, in a previous paper, see Dall'Aglio et al. [2016], we analysed those situations in which a governing majority does not correspond to a minimal winning coalition, i.e. it includes some parties, called non-critical, in the sense that, differently from the critical ones, they may recede from the majority, with no effect on the government. Our main interest focused on the matter that a non-critical party may become critical if other non-critical parties leave the majority; in this way, we introduced different orders of criticality. More precisely, we defined a party to be critical of order $k$ if it is necessary that at least $k-1$ other non-critical parties leave the majority without affecting it, before that its defection makes the coalition a losing one. It should be clear that the order of criticality of a party may be different in the various possible majorities it may belong to. In view of this, in this paper we want to associate to each party a unique value that aggregates the different orders of criticality that it has in the different winning coalitions.

The concept of criticality of the first order is the basis of the first power indices due to Shapley and Shubik Shapley and Shubik [1954] and Banzhaf Banzhaf [1965]. Other elements may influence the power of an agent; Myerson Myerson [1977] proposed to use an undirected graph, called communication structure, in order to represent the relationships among the agents, while Owen Owen [1977] introduced the a priori unions, or coalition structures, that account for existing agreements, not necessarily binding, among the agents. In the following years, Deegan and Packel Deegan and Packel [1978] and Holler Holler [1982] defined two new indices that consider only the minimal winning coalitions, i.e. those coalitions in which each agent is critical; Johnston Johnston [1978] used a similar model considering the quasi-minimal winning coalitions, i.e. those coalitions in which at least one agent is critical. Another important contribution is due to Kalai and Samet 
Kalai and Samet [1987] that added a weight to the elements characterizing each agent, besides the issue of criticality. Winter Winter [1989] extended Owen's idea of a priori unions by requiring that the different unions may join only according to a predefined scheme, that he called levels structure. In the years that followed, some papers dealt with situations in which only some coalitions are feasible, not only for communication reasons but also for possible incompatibilities among the agents; we refer to Algaba et al. [2003]; Algaba et al. [2004]; Bilbao et al. [1998]; Bilbao and Edelman [2000]; Gilles et al. [1992]; Brink Van den and Gilles [1996]; Brink Van den [1997]. Recently, in a monodimensional voting space, Amer and Carreras Amer R., Carreras [2001] considered the ideological distance of the agents and Fragnelli, Ottone and Sattanino Fragnelli et al. [2009] considered the issue of contiguity, while Chessa and Fragnelli Chessa and Fragnelli [2011] introduced the idea of connectedness in a possibly multidimensional voting space.

More in general, given a monotonic simple game, for each order of criticality, first, we introduce an appropriate criticality index aimed at measuring the number of times a player is critical of that order (and given the probabilities that various coalitions will form). Second, we propose a collective index aimed at aggregating the indices corresponding to different order of criticality into a unique value for each player. In order to compare our collective index with classical power indices, we analyse some monotonicity properties for weighted majority games Turnovec [1998] and we prove that within a given game, the behaviour of a collective index is coherent with the one of classical power indices, while a disaggregate representation of the criticality indices provides a more refined picture of the variation of each player's power across different games.

The paper is organized as follows. In the next section we provide some preliminary notions and notations. Section 3 is devoted to the definition of alternative indices of criticality and to the analysis of their basic properties. In Section 4 we study some monotonicity properties of a collective index in weighted majority games. Section 5 reconsiders the criticality indices in the light of the properties that characterize the Shapley-Shubik and Banzhaf indices. Section 6 presents some computational examples for the indices introduced in the previous sections. Section 7 concludes with some open problems.

\section{Preliminaries}

A cooperative game with transferable utility (TU-game) is a pair $(N, v)$, where $N=$ $\{1,2, \ldots, n\}$ denotes the finite set of players and $v: 2^{N} \rightarrow \mathbb{R}$ is the characteristic function, with $v(\varnothing)=0 . v(S)$ is the worth of coalition $S \subseteq N$, i.e. what players in $S$ may obtain standing alone.

A TU-game $(N, v)$ is simple when $v: 2^{N} \rightarrow\{0,1\}$, with $S \subseteq T \Rightarrow v(S) \leq v(T)^{\text {a }}$ and $v(N)=1$. If $v(S)=0$ then $S$ is a losing coalition, while if $v(S)=1$ then $S$ is

a This property is called monotonicity. 
a winning coalition. Given a winning coalition $S$, if $S \backslash\{i\}$ is losing then $i \in N$ is a critical player for $S$. When a coalition $S$ contains at least one critical player for it, $S$ is a quasi-minimal winning coalition; when all the players of $S$ are critical, it is a minimal winning coalition. A simple game may be defined also assigning the set of winning coalitions or the set of minimal winning coalitions.

A particular class of simple games is represented by the weighted majority games (w.m.g). A vector of weights $\left(w_{1}, w_{2}, \ldots, w_{n}\right)$ is associated to the players that leads to the following definition of the characteristic function of the corresponding weighted majority game $(N, w)$ :

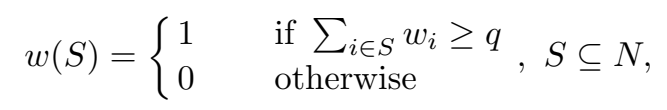

where $q$ is the majority quota. A weighted majority situation (w.m.s.) is often denoted as $\left[q ; w_{1}, w_{2}, \ldots, w_{n}\right]$. Usually, we ask that the game is proper or $N$-proper, i.e. if $S$ is winning then $N \backslash S$ is losing; for this aim it is sufficient to choose $q>\frac{1}{2} \sum_{i \in N} w_{i}$. Note that a simple game may not correspond to any weighted majority situation.

Given a TU-game $(N, v)$, an allocation is a $n$-dimensional vector $\left(x_{i}\right)_{i \in N} \in \mathbb{R}^{N}$ assigning to player $i \in N$ the amount $x_{i}$; an allocation $\left(x_{i}\right)_{i \in N}$ is efficient if $x(N)=$ $\sum_{i \in N} x_{i}=v(N)$. A solution is a function $\psi$ that assigns an allocation $\psi(v)$ to every TU-game $(N, v)$ belonging to a given class of games $\mathcal{G}$ with player set $N$.

For simple games, and in particular for weighted majority games, a solution is often called a power index, as each component $x_{i}$ may be interpreted as the percentage of power assigned to player $i \in N$. In the literature, several power indices were introduced; among others, we recall the following definitions.

The Shapley-Shubik index Shapley and Shubik [1954], $\phi$, is the natural version for simple games of the Shapley value Shapley [1953]. It is defined as the average of the marginal contributions of player $i$ w.r.t. all the possible orderings and it can be written as:

$$
\phi_{i}(v)=\sum_{S \subseteq N, S \ni i} \frac{(s-1) !(n-s) !}{n !} m_{i}(S), i \in N,
$$

where $n$ and $s$ denote the cardinalities of the set of players $N$ and of the coalition $S$, respectively and $m_{i}(S)=v(S)-v(S \backslash\{i\})$ denotes the marginal contribution of player $i \in N$ to coalition $S \subseteq N, S \ni i$.

The Banzhaf index Banzhaf [1965], $\beta$, is similar to the Shapley-Shubik index, but it considers the marginal contributions of a player to all possible coalitions, independently from the order of the players; first, we define:

$$
\beta_{i}(v)=\frac{1}{2^{n-1}} \sum_{S \subseteq N, S \ni i} m_{i}(S), i \in N .
$$

Consider the following properties: 
- Anonymity

Let $\pi$ be a permutation of the set of players $N$; a solution $\psi$ satisfies anonymity if $\psi_{i}(v)=\psi_{\pi(i)}(\pi v)$ for each $i \in N$, where $\pi v$ is the game in which $\pi v(S)=v(\pi(S))$ for each $S \subseteq N$.

- Null player

A player $i \in N$ is a null player if $v(S \cup\{i\})=v(S)$ for each $S \subseteq N \backslash\{i\}$; a solution $\psi$ satisfies null player property if $\psi_{i}=0$ for each null player $i \in N$.

- Transfer

A solution $\psi$ satisfies the transfer property if, given two games $v$ and $w$ with the same set of players $N$, then $\psi(v)+\psi(w)=\psi(v \vee w)+\psi(v \wedge w)$, where $v \vee w=\max \{v, w\}$ and $v \wedge w=\min \{v, w\}$, with $(v \vee w)(S)=$ $\max \{v(S), w(S)\}$ and $(v \wedge w)(S)=\min \{v(S), w(S)\}$ for each $S \subseteq N$.

- Banzhaf total power

A solution $\psi$ satisfies the Banzhaf total power property if $\sum_{i \in N} \psi_{i}=$ $\sum_{i \in N} \frac{1}{2^{n-1}} \sum_{S \subseteq N, S \ni i} m_{i}(S)$.

The Shapley-Shubik index is the unique efficient solution that satisfies the properties of Anonymity, Null player and Transfer Dubey [1975], while the Banzhaf index is characterized by the properties of Anonymity, Null player, Transfer and Banzhaf total power Dubey and Shapley [1979].

Given a simple game $(v, N)$, we reconsider the definition of criticality given in Dall'Aglio et al. [2016]:

Definition 1. Let $k \geq 0$ be an integer, let $M \subseteq N$, with $|M| \geq k+1$, be a winning coalition. We say that player $i$ is critical of the order $k+1$ for coalition $M$, via coalition $K$, with $|K|=k$ iff $K \subseteq M \backslash\{i\}$ is a set of minimal cardinality such that

$$
v(M \backslash K)-v(M \backslash(K \cup\{i\}))=1
$$

The meaning of the definition is that $K$ is a coalition of minimal cardinality such that $M \backslash K$ is still a winning coalition, while $M \backslash(K \cup\{i\})$ becomes a losing one $^{\mathrm{b}}$.

In the above definition, coalition $K$ is often omitted and we simply say that $i$ is critical of a certain order for coalition $M$.

Notice also that, when $k=0, K=\varnothing$, thus, a player is critical of order 1 if and only if it is critical in the usual sense.

Example 1. (Following the discussion in the Introduction) Consider two simple games $(N, u)$ and $(N, w)$ with $N=\{1,2\}$ and such that $u(\{1,2\})=1, u(\{1\})=$ $u(\{2\})=0$ and $w(\{1,2\})=w(\{1\})=w(\{2\})=1$. Both players 1 and 2 are critical of the first order for coalition $\{1,2\}$ in game $u$, while each player $i \in N$ is critical of

\footnotetext{
${ }^{\mathrm{b}}$ In other terms, $v(M \backslash T)=0$ or $v(M \backslash(T \cup\{i\})=1$ for any $T \subset M \backslash\{i\}$ with $|T|<k$.
} 
the first order for coalition $\{i\}$ in game $w$. Moreover, each player $i \in N$ is critical of the second order for coalition $\{1,2\}$ via the other player $j \neq i$ in game $w$.

Example 2. Consider a simple game $(N, v)$ with $N=\{1,2,3,4,5,6\}$ as the set of players and the following minimal winning coalitions (any superset of them is winning):

$$
\{1,2,3\} \quad\{1,2,4\} \quad\{1,2,5\} \quad\{1,3,4\} \quad\{1,3,5\}
$$

If we consider the winning coalition $M=\{1,2,3,4,5\}$, player 1 is critical of the first order, i.e. critical in the usual sense. Player $i=2,3$ is critical of the second order (consider $K=\{2,3\} \backslash\{i\}$ ), while player $i=4,5$ is critical of the third order (consider $K=\{2,4,5\} \backslash\{i\}$ or $K=\{3,4,5\} \backslash\{i\}$ ). If we consider $M=N$ the order of criticality for the first 5 players does not change, while player 6 is never critical: He cannot have any role in changing the status of a coalition.

The following results hold: i) Let $M \subseteq N$ be a winning coalition, then the players in $M$ may be partitioned according to their order of criticality, including the subset of those players that are never critical (Corollary 1 in Dall'Aglio et al. [2016]); ii) Let $i \in M$ be a player critical of the order $k+1, k \geq 1$ for coalition $M$, via coalition $K \subset M$; if a player $j \in K$ leaves the coalition, then $i$ is a player critical of the order $k$ for coalition $M \backslash\{j\}$, via coalition $K \backslash\{j\}$ (Proposition 3 in Dall'Aglio et al. [2016]); iii) If player $i \in M$ is critical of the order $k+1$ for coalition $M$, via coalition $K \subset M \backslash\{i\}$, then in $K$ there are at most $h-1$ players critical of order not greater than $h, h=1, \ldots, k$, otherwise $i$ should be critical of order at most $k$.

\section{Indices of criticality}

The aim of this section is to introduce an index for measuring the criticality of an agent. The starting point is the formula in Shapley and Shubik Shapley and Shubik [1954], that is based on the number of swings for each agent considering all the possible orderings of the agents. The motivation of this choice is to have an index that coincides with the Shapley-Shubik index when the criticality order is one.

The Shapley-Shubik index in a weighted majority game $v$ can be defined as the average number of times in which a player is critical w.r.t. a coalition when players enter the coalition in a random order. More formally, for any $i \in N$, let

$$
\phi_{i}(v)=\frac{1}{n !} \sum_{\pi \in \Pi} \sigma_{i}(\pi)
$$

where $\pi$ is any permutation of $N, \Pi$ is the class of all permutations and

$$
\sigma_{i}(\pi)=\left\{\begin{array}{l}
1 \text { if player } i \text { is critical in } P_{\pi}^{i} \cup\{i\} \\
0 \text { otherwise }
\end{array}\right.
$$

and $P_{\pi}^{i}$ is the set of players in $N$ that precede $i$ in the order $\pi$. 
We may, therefore, introduce an index which measures for any player $i$ his power in being $k$ order critical, with $k=1,2, \ldots, n$.

$$
\phi_{i, k}(v)=\frac{1}{n !} \sum_{\pi \in \Pi} \sigma_{i, k}(\pi)
$$

with

$$
\sigma_{i, k}(\pi)=\left\{\begin{array}{l}
1 \text { if } i \text { is critical of order } k \text { in } P_{\pi} \cup\{i\} \\
0 \text { otherwise }
\end{array}\right.
$$

With simple combinatorial arguments, we may write these indices in a way that is familiar to anybody working with the Shapley-Shubik index:

$$
\phi_{i, k}(v)=\sum_{S \ngtr i} \frac{|S| !(n-|S|-1) !}{n !} d c_{k}(i, S \cup\{i\})
$$

where

$$
d c_{k}(i, M)=\left\{\begin{array}{l}
1 \text { if } i \text { is critical of order } k \text { in the winning coalition } M \\
0 \text { otherwise }
\end{array}\right.
$$

We may therefore associate a whole distribution of indices of criticality to a player:

$$
\Phi_{i}(v)=\left(\phi_{i, 1}(v) \phi_{i, 2}(v), \ldots, \phi_{i, n}(v)\right)
$$

Example 1. It is immediate to verify that: $\Phi_{1}(u)=\Phi_{2}(u)=\left(\frac{1}{2}, 0\right)$ and $\Phi_{1}(w)=$ $\Phi_{2}(w)=\left(\frac{1}{2}, \frac{1}{2}\right)$

Example 2. With the aid of Mathematica, we computed the distribution of indices for every player:

$$
\begin{gathered}
\Phi_{1}(v)=\left(\frac{17}{30}, 0,0,0,0,0\right) \quad \Phi_{2}(v)=\Phi_{3}(v)=\left(\frac{3}{20}, \frac{3}{10}, 0,0,0,0\right) \\
\Phi_{4}(v)=\Phi_{5}(v)=\left(\frac{1}{15}, \frac{3}{20}, \frac{1}{5}, 0,0,0\right) \quad \Phi_{6}(v)=(0,0,0,0,0,0)
\end{gathered}
$$

Recalling the efficiency property for the Shapley-Shubik index, we have

$$
\sum_{i \in N} \phi_{i, 1}(v)=\sum_{i \in N} \phi_{i}(v)=v(N)=1
$$

Some bounds can be established.

Proposition 1. The following inequalities hold

$$
1 \leq \sum_{k=1}^{n} \sum_{i \in N} \phi_{i, k}(v) \leq n
$$

and these are sharp, i.e., they can be attained. 
8 Dall'Aglio M., Fragnelli V., Moretti S.

Proof. Clearly, by (10)

$$
\sum_{k=1}^{n} \sum_{i \in N} \phi_{i, k}(v) \geq \sum_{i \in N} \phi_{1, k}(v)=1
$$

To verify that the bound can be attained, consider the following game

$$
\underline{v}(S)=\left\{\begin{array}{l}
1 \text { if } S=N \\
0 \text { otherwise }
\end{array}\right.
$$

Clearly, the players are symmetrical and no player can be critical beyond the first order. Thus

$$
\Phi_{i}(\underline{v})=\left(\frac{1}{n}, 0, \ldots, 0\right)
$$

To prove the second inequality, note that no player can be critical of more than one order. Therefore, for any permutation $\pi \in \Pi$,

$$
\sum_{k=1}^{n} \sum_{i \in N} \sigma_{i, k}(\pi) \leq n
$$

Thus, recalling the definition (5),

$$
\sum_{k=1}^{n} \sum_{i \in N} \phi_{i, k}(v)=\sum_{k=1}^{n} \sum_{i \in N} \frac{1}{n !} \sum_{\pi \in \Pi} \sigma_{i, k}(\pi)=\frac{1}{n !} \sum_{\pi \in \Pi} \sum_{k=1}^{n} \sum_{i \in N} \sigma_{i, k}(\pi) \leq n
$$

the inequality deriving from (15).

The following game achieves the upper bound

$$
\bar{v}(S)=\left\{\begin{array}{l}
0 \text { if } S=\varnothing \\
1 \text { otherwise }
\end{array}\right.
$$

Now, in each permutation $\pi$ of $N$, player $\pi(1)$ is critical of the first order, player $\pi(2)$ is critical of the second order, player $\pi(3)$ is critical of order 3 , and so on. Therefore,

$$
\Phi_{i}(v)=\left(\frac{1}{n}, \ldots, \frac{1}{n}\right)
$$

and the upper bound is reached.

In the example ??, player 6 exhibits null values because it is always non-critical. To point out the role of non-critical players, we may introduce a suitable index:

$$
\phi_{i, N C}(v)=\sum_{S \not i} \frac{|S| !(n-|S|-1) !}{n !} d c_{N C}(i, S \cup\{i\})
$$

where

$$
d c_{N C}(i, M)=\left\{\begin{array}{l}
1 \text { if } i \text { is never critical in the winning coalition } M \\
0 \text { otherwise }
\end{array}\right.
$$


Example 2. Again, using Mathematica, we obtain:

$$
\phi_{1, N C}(v)=\phi_{2, N C}(v)=\phi_{3, N C}(v)=\phi_{4, N C}(v)=\phi_{5, N C}(v)=0 \quad \phi_{6, N C}(v)=\frac{23}{60}
$$

The Shapley-Shubik index of power is naturally paired with the (nonnormalized) Bahnzaf index. This index counts the proportion of times that a certain player is critical w.r.t. any coalition that includes him. It is thus natural to define a Bahnzaf index of criticality of any order:

$$
\beta_{i, k}(v)=\sum_{S \not i} \frac{d c_{k}(i, S \cup\{i\})}{2^{n-1}}
$$

Also in this case we have a distribution of indices:

$$
B_{i}(v)=\left(\beta_{i, 1}(v) \beta_{i, 2}(v), \ldots, \beta_{i, n}(v)\right)
$$

and an index of non-criticality

$$
\beta_{i, N C}(v)=\sum_{S \ngtr i} \frac{d c_{N C}(i, S \cup\{i\})}{2^{n-1}}
$$

Now, we return to the introductory examples.

Example 1. Since the weights in (7) and (21) coincide when $n=2$, then we have $B_{1}(u)=B_{2}(u)=\left(\frac{1}{2}, 0\right)$ and $B_{1}(w)=B_{2}(w)=\left(\frac{1}{2}, \frac{1}{2}\right)$.

Example 2. Here, the values differ from the Shapley-derived:

$$
\begin{aligned}
& B_{1}(v)=\left(\frac{5}{8}, 0,0,0,0,0\right) \quad B_{2}(v)=B_{3}(v)=\left(\frac{1}{4}, \frac{3}{16}, 0,0,0,0\right) \\
& B_{4}(v)=B_{5}(v)=\left(\frac{1}{8}, \frac{3}{16}, \frac{1}{16}, 0,0,0\right) \quad B_{6}(v)=(0,0,0,0,0,0)
\end{aligned}
$$

and

$$
B_{1, N C}(v)=B_{2, N C}(v)=B_{3, N C}(v)=B_{4, N C}(v)=B_{5, N C}(v)=0 \quad B_{6, N C}(v)=\frac{5}{16}
$$

The indices of power provide an easy-to-use and easy-to-understand means to compare the capability of players in being decisive, i.e. critical in forming a winning coalition. When we extend these notions by including higher order of criticality, we need to compare distributions (i.e. vectors) of indices. In what follows, we provide numeric indices that synthesize the whole distribution and allow the comparison of power among players.

Definition 2. The Collective Shapley-Shubik (CSS) power index through all orders of criticality for player $i \in N$ is defined by

$$
\bar{\Phi}_{i}(v)=\frac{\sum_{h=1}^{n} \phi_{i, h}(v) h^{-1}}{\sum_{h=1}^{n} h^{-1}}
$$


Similarly, the Collective Banzhaf (CB) power index through all orders of criticality for the same player is

$$
\bar{B}_{i}(v)=\frac{\sum_{h=1}^{n} \beta_{i, h}(v) h^{-1}}{\sum_{h=1}^{n} h^{-1}}
$$

Those two indices range between 0 and 1 , but no result concerning their total power - something similar to the efficiency property for Shapley-Shubik index, or the Bahnzaf total power, is currently known ${ }^{\mathrm{c}}$. Their importance lies in their ability to compare the power of different players. Let us denote as $\succeq_{C S S}\left(\succeq_{C B}\right.$, resp.) the order established among the players by the Collective Shapley-Shubik (Collective Bahnzaf, resp.) index.

Example 1. By relation (24), it is easy to calculate the collective Shapley index for the two players in the games $u$ and $w$, we have: $\bar{\Phi}_{1}(u)=\bar{\Phi}_{2}(u)=\frac{1}{3}$ and $\bar{\Phi}_{1}(w)=\bar{\Phi}_{2}(w)=\frac{1}{2}$. Notice that the collective Shapley index emphasizes the different role of the two players over the games $u$ and $w$ (while the Shapley-Shubik index is the same in the two games). Identical conclusions hold for $C B$.

Example 2. Clearly

$$
\begin{aligned}
& \bar{\Phi}_{1}(v)=\frac{34}{147} \quad \bar{\Phi}_{2}(v)=\bar{\Phi}_{3}(v)=\frac{6}{49} \\
& \bar{\Phi}_{4}(v)=\bar{\Phi}_{5}(v)=\frac{25}{294} \quad \bar{\Phi}_{6}(v)=0
\end{aligned}
$$

and

$$
\begin{aligned}
& \bar{B}_{1}(v)=\frac{25}{98} \quad \bar{B}_{2}(v)=\bar{B}_{3}(v)=\frac{55}{392} \\
& \bar{B}_{4}(v)=\bar{B}_{5}(v)=\frac{115}{1176} \quad \bar{B}_{6}(v)=0
\end{aligned}
$$

Clearly,

$$
1 \succ_{C S S} 2 \sim_{C S S} 3 \succ_{C S S} 4 \sim_{C S S} 5 \succ_{C S S} 6,
$$

and the same order applies through the Collective Bahnzaf index.

\section{Monotonicity properties for weighted majority games}

As we said in the Preliminaries, a particular class of simple games is represented by the weighted majority games.

When it comes to measuring power in committees or parliaments, most often a party's power is not perfectly proportional to its size. However, if no impediment

c The lower bound is obvious, all the quantities being non-negative; as both $\phi_{i, h}(v)$ and $\beta_{i, h}(v)$ are never larger than 1 , for any $i$ and $h$, then the numerators in both (24) and (25) are not greater than the denominators, so the upper bound holds. 
occurs, the rankings in power and in size should agree within the same assembly and across different assemblies, when the weights clearly show the advantage of a party over the others. These situations are captured by the following properties:

Definition 3. Let $\left[q ; w_{1}, \ldots, w_{n}\right]$ be a w.m.s. and let $v_{m}$ be the corresponding game. A power index $\psi: \mathcal{W}(N) \rightarrow \mathbb{R}_{+}^{n}$ is locally monotone whenever $w_{i}>w_{j}$ implies

$$
\psi_{i}\left(v_{m}\right) \geq \psi_{j}\left(v_{m}\right)
$$

Consider now two weighted majority situations $\left[q ; w_{1}, \ldots, w_{n}\right]$ and $\left[q ; w_{1}^{\prime}, \ldots, w_{n}^{\prime}\right]$, with

$$
\sum_{i \in N} w_{i}=\sum_{i \in N} w_{i}^{\prime}=C,
$$

Let $v_{m}$ and $v_{m}^{\prime}$ be the corresponding games. The power index $\psi$ is globally monotone if

$$
w_{i^{*}}>w_{i^{*}}^{\prime} \quad \text { and } \quad w_{j} \leq w_{j}^{\prime} \text { for all } j \neq i^{*}
$$

implies

$$
\psi_{i^{*}}\left(v_{m}\right) \geq \psi_{i^{*}}\left(v_{m}^{\prime}\right)
$$

Turnovec Turnovec [1998] shows that the (first order) Shapley-Shubik power index is both locally and globally monotonic. The same properties apply to the (non-normalized) Banzhaf power index. We now extend these properties to the Collective indices just introduced.

Adapting Turnovec Turnovec [1998], we define the sets

$$
C_{i k s}\left(v_{m}\right):=\{S \subset N: i \text { is critical of order } k \text { w.r.t. } S \text { and }|S|=s\}
$$

and

$$
\mathfrak{C}_{i k s}\left(v_{m}\right):=\bigcup_{\ell \leq k} C_{i \ell s}\left(v_{m}\right)
$$

We can now write the Shapley-Shubik index of criticality of any order $k$ as

$$
\phi_{i, k}\left(v_{m}\right)=\sum_{s=1}^{n} \frac{(s-1) !(n-s) !}{n !}\left|C_{i k s}\left(v_{m}\right)\right|
$$

where, as usual, $|\cdot|$ denotes the cardinality of a set.

A similar expression is found for the CSS index

$$
\bar{\Phi}_{i}\left(v_{m}\right)=\sum_{s=1}^{n} \frac{(s-1) !(n-s) !}{n !}\left(\frac{\sum_{h=1}^{n}\left|C_{i h s}\left(v_{m}\right)\right| h^{-1}}{\sum_{h=1}^{n} h^{-1}}\right)
$$

Simpler expressions hold for the Bahnzaf-type indices

$$
\beta_{i, k}\left(v_{m}\right)=\sum_{s=1}^{n} \frac{\left|C_{i k s}\left(v_{m}\right)\right|}{2^{n-1}}
$$


and

$$
\bar{B}_{i}\left(v_{m}\right)=\sum_{s=1}^{n} \frac{1}{2^{n-1}}\left(\frac{\sum_{h=1}^{n}\left|C_{i h s}\left(v_{m}\right)\right| h^{-1}}{\sum_{h=1}^{n} h^{-1}}\right)
$$

We begin by showing the local monotonicity of the indices.

Lemma 1. Consider a w.m.s. $\left[q ; w_{1}, \ldots, w_{n}\right]$, and the associated game $v_{m}$. Then, $w_{i}>w_{j}$ implies

$$
\left|\mathfrak{C}_{i k s}\left(v_{m}\right)\right| \geq\left|\mathfrak{C}_{j k s}\left(v_{m}\right)\right| \quad \text { for any } k, s=1, \ldots, n .
$$

Proof. Assume $w_{i}>w_{j}$. We now show that to any $S \in C_{j k s}\left(v_{m}\right)$ there corresponds a set $S^{\prime} \in C_{i \ell s}\left(v_{m}\right)$ with $\ell \leq k$. Since no pair of sets $S^{\prime}$ coincide, this proves (36).

Since $S \in C_{j k s}\left(v_{m}\right)$, then $|S|=s$, and there exists $K \subset S \backslash\{j\},|K|=k-1$, such that

$$
v_{m}(S \backslash K)=1 \quad v_{m}(S \backslash(K \cup\{j\}))=0
$$

and the $K$ is the set of minimal cardinality satisfying (37). Denoting $\tilde{K}=S \backslash(K \cup$ $\{j\})$, we may restate $(37)$ as

$$
v_{m}(\tilde{K} \cup\{j\})=1 \quad v_{m}(\tilde{K})=0
$$

We now consider several cases

Case 1. $i \in K \subset S$. We show that $i$ is critical of order $k$ or lower for the same coalition $S$ by showing that an analogous of (37), or, equivalently, (38), holds for $S$, $i$ and $K^{\prime}=K \cup\{j\} \backslash\{i\}$. We do not need to check whether this is a set of minimal cardinality, as long as we do not care about the exact order of criticality for player $i$.

Now $S=K^{\prime} \cup \tilde{K} \cup\{i\}$,

$$
v_{m}\left(S \backslash K^{\prime}\right)=v_{m}(\tilde{K} \cup\{i\}) \geq v_{m}(\tilde{K} \cup\{j\})=1,
$$

by (38), with the inequality justified by the assumption $w_{i} \geq w_{j}$. Moreover, by (38) again,

$$
v_{m}\left(S \backslash\left(K^{\prime} \cup\{i\}\right)=v_{m}(\tilde{K})=0 .\right.
$$

Case 2. $i \in S \backslash K$. We show that $i, S$ and $K$ satisfy an analogous of condition (38). This time $S=K \cup \tilde{K}^{\prime} \cup\{i\}$ with $\tilde{K}^{\prime}=\tilde{K} \cup\{j\} \backslash\{i\}$. Now (38) and the assumption imply,

$$
v_{m}(S \backslash K)=v_{m}\left(\tilde{K}^{\prime} \cup\{i\}\right)=v_{m}(\tilde{K} \cup\{j\})=1
$$

and

$$
v_{m}(S \backslash(K \cup\{i\}))=v_{m}\left(\tilde{K}^{\prime}\right)=v_{m}(\tilde{K} \cup\{j\} \backslash\{i\}) \leq v_{m}(\tilde{K})=0 .
$$

Case 3. $i \notin S$. We now show that $i$ is critical of order $k$ or lower w.r.t. coalition $S^{\prime}=S \cup\{i\} \backslash\{j\}$ of size $s$. Here $S^{\prime}=K \cup \tilde{K} \cup\{i\}$, while conditions (38) imply:

$$
v_{m}\left(S^{\prime} \backslash K\right)=v_{m}(\tilde{K} \cup\{i\}) \geq v_{m}(\tilde{K} \cup\{j\})=1
$$


and

$$
v_{m}\left(S^{\prime} \backslash(K \cup\{i\})\right)=v_{m}(\tilde{K})=0 .
$$

Once again, player $i$ is critical of order $k$ or lower for a coalition of cardinality $s$

This leads to

Theorem 1. Both CSS and CB are locally monotonic

Proof. If $w_{i}>w_{j}$, Lemma 1 shows that

$$
\frac{\sum_{h=1}^{n}\left|C_{i h s}\left(v_{m}\right)\right| h^{-1}}{\sum_{h=1}^{n} h^{-1}} \geq \frac{\sum_{h=1}^{n}\left|C_{j h s}\left(v_{m}\right)\right| h^{-1}}{\sum_{h=1}^{n} h^{-1}} \quad \text { for any } s=1, \ldots, n,
$$

and, therefore, that $\bar{\Phi}_{i}\left(v_{m}\right) \geq \bar{\Phi}_{j}\left(v_{m}\right)$ and $\bar{B}_{i}\left(v_{m}\right) \geq \bar{B}_{j}\left(v_{m}\right)$.

To verify the global monotonicity of the same indices, we need to compare the two w.m.s. $\left[q ; w_{1}, \ldots, w_{n}\right]$ and $\left[q ; w_{1}^{\prime}, \ldots, w_{n}^{\prime}\right]$, and the corresponding games, $v_{m}$ and $v_{m}^{\prime}$ under (28) and (29). The first Lemma is, in its essence, a reworking of a result by Turnovec.

Lemma 2 (Turnovec Turnovec [1998], Lemma 5). Suppose (28 and (29) hold. Then for any $P \subset N \backslash\left\{i^{*}\right\}$ we have

$$
v_{m}\left(P \cup\left\{i^{*}\right\}\right) \geq v_{m}^{\prime}\left(P \cup\left\{i^{*}\right\}\right) \quad \text { and } \quad v_{m}(P) \leq v_{m}^{\prime}(P)
$$

Proof. By (28) we can write

$$
w_{i^{*}}=C-\sum_{j \neq i^{*}} w_{j} \quad w_{i^{*}}^{\prime}=C-\sum_{j \neq i^{*}} w_{j}^{\prime}
$$

and by (29) we have

$$
w_{i^{*}}-w_{i^{*}}^{\prime}=\sum_{j \neq i^{*}}\left(w_{j}^{\prime}-w_{j}\right) \geq \sum_{j \in P}\left(w_{j}^{\prime}-w_{j}\right) \geq 0 .
$$

Therefore,

$$
w_{i^{*}}+\sum_{j \in P} w_{j} \geq w_{i^{*}}^{\prime}+\sum_{j \in P} w_{j}^{\prime}
$$

and

$$
\sum_{j \in P} w_{j} \leq \sum_{j \in P} w_{j}^{\prime}
$$

which leads to (42)

To establish global monotonicity we need another Lemma.

Lemma 3. Assumptions (28) and (29) imply

$$
\left|\mathfrak{C}_{i^{*} k s}\left(v_{m}\right)\right| \geq\left|\mathfrak{C}_{i^{*} k s}\left(v_{m}^{\prime}\right)\right| \quad \text { for any } k, s=1, \ldots, n \text {. }
$$


Proof. We will show that, if $S \in C_{i^{*} k s}\left(v_{m}^{\prime}\right)$, then $S \in \mathfrak{C}_{i^{*} \ell s}\left(v_{m}\right)$ for some $\ell \leq k$. In fact, for such a coalition $S$ we have that there exists $K \subset S \backslash\left\{i^{*}\right\},|K|=k-1$ such that

$$
v_{m}^{\prime}(S \backslash K)=1 \quad \text { and } \quad v_{m}^{\prime}\left(S \backslash\left(K \cup\left\{i^{*}\right\}\right)\right)=0
$$

and any $T \subset S \backslash\left\{i^{*}\right\}$ with cardinality lower than $k-1$ does not satisfy both equations.

A straightforward application of Lemma 2 yields

$$
v_{m}(S \backslash K) \geq v_{m}^{\prime}(S \backslash K)=1
$$

and

$$
v_{m}\left(S \backslash\left(K \cup\left\{i^{*}\right\}\right)\right) \leq v_{m}^{\prime}\left(S \backslash\left(K \cup\left\{i^{*}\right\}\right)\right)=0
$$

and, therefore, concludes the proof.

This leads to

Theorem 2. Both CSS and CB are globally monotonic.

Proof. If assumptions (28) and (29) hold, Lemma 3 shows that

$$
\frac{\sum_{h=1}^{n}\left|C_{i^{*} h s}\left(v_{m}\right)\right| h^{-1}}{\sum_{h=1}^{n} h^{-1}} \geq \frac{\sum_{h=1}^{n}\left|C_{i^{*} h s}\left(v_{m}^{\prime}\right)\right| h^{-1}}{\sum_{h=1}^{n} h^{-1}} \quad \text { for any } s=1, \ldots, n,
$$

and, therefore, that $\bar{\Phi}_{i}^{*}\left(v_{m}\right) \geq \bar{\Phi}_{i}^{*}\left(v_{m}^{\prime}\right)$ and $\bar{B}_{i}^{*}\left(v_{m}\right) \geq \bar{B}_{i}^{*}\left(v_{m}^{\prime}\right)$.

Remark 1. Turnovec Turnovec [1998] shows (Theorem 4) that, under suitable symmetry assumptions, global monotonicity implies local monotonicity for power indices. Whether this fact applies here as well remains an open question.

\section{First steps toward a characterization}

We may ask which among the properties that characterize the indices of ShapleyShubik and Banzhaf may be used for characterizing the criticality indices. We begin with an easy result.

Proposition 2. The criticality indices $\Phi, \bar{\Phi}, B$ and $\bar{B}$ satisfy anonymity and null player properties.

Proof. It is straigthforward, after noticing that relations (7) and (21) satisfy the two properties.

As previously noted, no property concerning the efficiency or the total power is known for these indices. Also the transfer property is not satisfied, as the following example shows. 
Example 3. Consider the following example where $N=\{1,2,3\}, v$ is the simple game whose unique minimal winning coalition is $\{1,2\}$ and $w$ is the simple game whose minimal winning coalitions are $\{1,3\},\{2,3\}$. In this case, we have:

\begin{tabular}{l|cccc} 
index/game & $v$ & $w$ & $v \vee w$ & $v \wedge w$ \\
\hline$\Phi_{1}$ & $\left(\frac{1}{2}, 0,0\right)$ & $\left(\frac{1}{6}, \frac{1}{3}, 0\right)$ & $\left(\frac{1}{3}, \frac{1}{3}, 0\right)$ & $\left(\frac{1}{3}, 0,0\right)$ \\
$\Phi_{2}$ & $\left(\frac{1}{2}, 0,0\right)$ & $\left(\frac{1}{6}, \frac{1}{3}, 0\right)$ & $\left(\frac{1}{3}, \frac{1}{3}, 0\right)$ & $\left(\frac{1}{3}, 0,0\right)$ \\
$\Phi_{3}$ & $(0,0,0)$ & $\left(\frac{2}{3}, 0,0\right)$ & $\left(\frac{1}{3}, \frac{1}{3}, 0\right)$ & $\left(\frac{1}{3}, 0,0\right)$ \\
$\bar{\Phi}$ & $\left(\frac{3}{11}, \frac{3}{11}, 0\right)$ & $\left(\frac{2}{11}, \frac{2}{11}, \frac{4}{11}\right)$ & $\left(\frac{3}{11}, \frac{3}{11}, \frac{3}{11}\right)$ & $\left(\frac{2}{11}, \frac{2}{11}, \frac{2}{11}\right)$ \\
\hline$B_{1}$ & $\left(\frac{1}{2}, 0,0\right)$ & $\left(\frac{1}{4}, \frac{1}{4}, 0\right)$ & $\left(\frac{1}{2}, \frac{1}{4}, 0\right)$ & $\left(\frac{1}{4}, 0,0\right)$ \\
$B_{2}$ & $\left(\frac{1}{2}, 0,0\right)$ & $\left(\frac{1}{4}, \frac{1}{4}, 0\right)$ & $\left(\frac{1}{2}, \frac{1}{4}, 0\right)$ & $\left(\frac{1}{4}, 0,0\right)$ \\
$B_{3}$ & $(0,0,0)$ & $\left(\frac{3}{4}, 0,0\right)$ & $\left(\frac{1}{2}, \frac{1}{4}, 0\right)$ & $\left(\frac{1}{4}, 0,0\right)$ \\
$\bar{B}$ & $\left(\frac{3}{11}, \frac{3}{11}, 0\right)$ & $\left(\frac{9}{44}, \frac{9}{44}, \frac{9}{22}\right)$ & $\left(\frac{15}{44}, \frac{15}{44}, \frac{15}{44}\right)$ & $\left(\frac{3}{22}, \frac{3}{22}, \frac{3}{22}\right)$
\end{tabular}

It is easy to check that the transfer property does not hold, as in $v \vee w$ and $v \wedge w$ the players are symmetric, while in $v$ and $w$ they are not.

More generally, the following result holds.

Proposition 3. The transfer property is not satisfied by any weighted average of the Shapley-Shubik (Banzhaf resp.) indices of criticalities of all orders, with strictly positive weights.

Proof. Referring to Example 3, notice that $\Phi_{3}(v)+\Phi_{3}(w)=\left(\frac{2}{3}, 0,0\right)$ and $\Phi_{3}(v \vee$ $w)+\Phi_{3}(w \wedge w)=\left(\frac{2}{3}, \frac{1}{3}, 0\right)$ and no set of posistive weights can equate the averages of the two distributions.

In view of this, we need other properties for characterizing the criticality indices.

\section{Examples}

Consider Example 3 in Dall'Aglio, Fragnelli, Moretti (2016). In the weighted majority situation $[51 ; 44,3,3,3,3,3,3]$, party 1 is critical of order 1 , while the other six parties are critical of order 4 and there are no parties critical of order 2 or 3 . If we account the ordering (we write the weights of the parties instead of their numbers):

$$
44,3,3,3,3,3,3
$$

the fourth party is critical of order 1, the fifth party is critical of order 2 via the fourth party (or the second, or the third), the sixth party is critical of order 3 via the fourth and the fifth parties (or any pair of parties from the second to the fifth), the seventh party is critical of order 4 via the fourth, the fifth and the sixth parties 
(or any triple of parties from the second to the sixth). The same reasoning applies to all the permutations of the parties with weight 3 .

Accounting the ordering:

$$
3,3,3,3,44,3,3
$$

the fifth party is critical of order 1 , the sixth party is critical of order 3 , the seventh party is critical of order 4. Again, the same reasoning applies to all the permutations of the parties with weight 3 .

If we consider all the possible orderings we have Table 1, where the Roman number below each party after the critical one denotes its order of criticality.

Table 1. Orders of criticality for each ordering of the parties in $[51 ; 44,3,3,3,3,3,3]$.

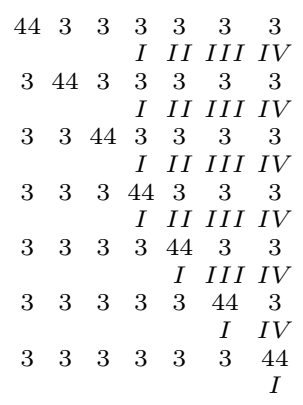

In terms of distributions, we have

$$
\begin{aligned}
& \Phi_{1}=\left(\frac{24}{42}, 0,0,0,0,0,0\right) \quad \Phi_{2}=\cdots=\Phi_{7}=\left(\frac{3}{42}, \frac{4}{42}, \frac{5}{42}, \frac{6}{42}, 0,0,0\right) \\
& \bar{\Phi}=\left(\frac{80}{363}, \frac{3}{40}, \ldots, \frac{3}{40}\right) \\
& B_{1}=\left(\frac{21}{32}, 0,0,0,0,0,0\right) \quad B_{2}=\cdots=B_{7}=\left(\frac{5}{32}, \frac{5}{32}, \frac{5}{64}, \frac{1}{64}, 0,0,0\right) \\
& \bar{B}=\left(\frac{245}{968}, \frac{1}{10}, \ldots, \frac{1}{10}\right)
\end{aligned}
$$

Now, consider Example 1 in Dall'Aglio, Fragnelli, Moretti (2016). In the weighted majority situation $[51 ; 40,8,5,5,5]$, party 1 is critical of order 1 , while the other four parties are critical of order 2 . If we consider all the possible orderings we have Table 2 .

In terms of distributions, we have

$$
\Phi_{1}=\left(\frac{1}{2}, 0,0,0,0\right) \quad \Phi_{2}=\left(\frac{1}{4}, \frac{1}{5}, 0,0,0\right) \quad \Phi_{3}=\Phi_{4}=\Phi_{5}=\left(\frac{1}{12}, \frac{3}{10}, 0,0,0\right)
$$


Table 2. Orders of criticality for each ordering of the parties in $[51 ; 40,8,5,5,5]$.

$$
\begin{aligned}
& \begin{array}{lllll}
40 & 8 & 5 & 5 & 5 \\
& & I & I I & I I
\end{array} \\
& \begin{array}{lllll}
40 & 5 & 8 & 5 & 5
\end{array} \\
& \text { I II II } \\
& \begin{array}{ccccc}
40 & 5 & 5 & 8 & 5 \\
& & & I & I I
\end{array} \\
& \begin{array}{lllll}
40 & 5 & 5 & 5 & 8
\end{array} \\
& \begin{array}{llllc}
8 & 40 & 5 & 5 & 5
\end{array} \\
& \text { I } I I \text { II } \\
& \begin{array}{lllll}
5 & 40 & 8 & 5 & 5
\end{array} \\
& \text { I II II } \\
& \begin{array}{lllll}
5 & 40 & 5 & 8 & 5
\end{array} \\
& \begin{array}{lll}
5 & I I
\end{array} \\
& \begin{array}{lllll}
5 & 40 & 5 & 5 & 8 \\
& & & I & I I
\end{array} \\
& \begin{array}{lllll}
8 & 5 & 40 & 5 & 5
\end{array} \\
& \text { I II II } \\
& \begin{array}{ccccc}
5 & 8 & 40 & 5 & 5 \\
& & I & I I & I I
\end{array} \\
& 5540 \quad 8 \quad 5 \\
& \begin{array}{lc}
8 & 5 \\
I & I I
\end{array}
\end{aligned}
$$

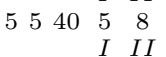

$$
\begin{aligned}
& \begin{array}{lllll}
8 & 5 & 5 & 40 & 5
\end{array} \\
& \begin{array}{lllcc}
5 & 8 & 5 & 4 & I I \\
& 40 & 5
\end{array} \\
& \begin{array}{lll}
I & I I
\end{array} \\
& \begin{array}{lllll}
5 & 5 & 8 & 40 & 5
\end{array} \\
& \text { I II } \\
& \begin{array}{ccccc}
5 & 5 & 5 & 40 & 8 \\
& & I & I I
\end{array} \\
& \begin{array}{lllll}
8 & 5 & 5 & 5 & 40
\end{array} \\
& \begin{array}{lllll}
5 & 8 & 5 & 5 & 40
\end{array} \\
& \begin{array}{ccccc}
5 & 5 & 8 & 5 & 40 \\
& & & & I
\end{array} \\
& \begin{array}{lllll}
5 & 5 & 5 & 8 & 40 \\
& & &
\end{array} \\
& \bar{\Phi}=\left(\frac{30}{137}, \frac{21}{137}, \frac{14}{137}, \frac{14}{137}, \frac{14}{137}\right) \\
& B_{1}=\left(\frac{1}{2}, 0,0,0,0\right) \quad B_{2}=\left(\frac{3}{8}, \frac{1}{16}, 0,0,0\right) \quad B_{3}=B_{4}=B_{5}=\left(\frac{1}{8}, \frac{3}{16}, 0,0,0\right) \\
& \bar{B}=\left(\frac{30}{137}, \frac{195}{1096}, \frac{105}{1096}, \frac{105}{1096}, \frac{105}{1096}\right)
\end{aligned}
$$

Now, consider the weighted majority situation $[12 ; 10,2,2,1,1]$, party 1 is critical of order 1, while the other four parties are critical of order 3 and there are no parties critical of order 2 . If we consider all the possible orderings we have Table 3 . 
Table 3. Orders of criticality for each ordering of the parties in $[12 ; 10,2,2,1,1]$.

$$
\begin{aligned}
& \begin{array}{ccccc}
10 & 2 & 2 & 1 & 1 \\
& I & I I & 0 & I I I
\end{array} \\
& \begin{array}{ccccc}
10 & 2 & 1 & 2 & 1 \\
& I & 0 & I I & I I I
\end{array} \\
& \begin{array}{lllll}
10 & 2 & 1 & 1 & 2 \\
& I & 0 & \text { II } & \text { III }
\end{array} \\
& \begin{array}{lllll}
0 & 1 & 2 & 2 & 1
\end{array} \\
& \begin{array}{lllll}
10 & 1 & 2 & 1 \\
& & \text { I } & \text { II } & \text { III }
\end{array} \\
& \begin{array}{ccccc}
10 & 1 & 2 & 1 & 2 \\
& & I & I I & I I I
\end{array} \\
& \begin{array}{lllll}
10 & 1 & 1 & 2 & 2
\end{array} \\
& \begin{array}{llll}
10 & 2 & 1 & 1
\end{array} \\
& \begin{array}{cccc}
10 & 2 & 1 & 1 \\
I & I I & 0 & I I I
\end{array} \\
& \begin{array}{ccccc}
2 & 10 & 1 & 2 & 1 \\
& I & 0 & I I & I I I
\end{array} \\
& \begin{array}{cccc}
10 & 1 & 1 & 2 \\
I & 0 & I I & I I I
\end{array}
\end{aligned}
$$

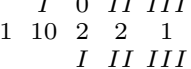

$$
\begin{aligned}
& \begin{array}{llllr}
1 & 10 & 2 & 1 & 2
\end{array}
\end{aligned}
$$

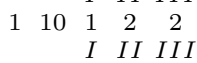

$$
\begin{aligned}
& 221110 \\
& \begin{array}{llllc}
2 & 1 & 2 & 1 & I \\
& & & & I
\end{array}
\end{aligned}
$$

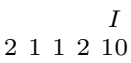

$$
\begin{aligned}
& I \\
& \begin{array}{rrrr}
12 & 211 \\
& & & 1
\end{array} \\
& \begin{array}{llll}
12 & 2 & 2 & 10 \\
& & & I
\end{array}
\end{aligned}
$$

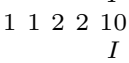

$$
\begin{aligned}
& \begin{array}{ccccc}
2 & 2 & 10 & 1 & 1 \\
& & 0 & \text { III }
\end{array} \\
& \begin{array}{lllll}
2 & 1 & 10 & 2 & 1
\end{array} \\
& \begin{array}{lcc}
I & \text { II } & \text { III }
\end{array} \\
& \begin{array}{lllll}
2 & 1 & 10 & 1 & 2 \\
& & I & \text { II } & \text { III }
\end{array} \\
& \begin{array}{lllll}
1 & 2 & 10 & 2 & 1
\end{array} \\
& \text { I III III } \\
& \begin{array}{ccccc}
1 & 2 & 10 & 1 & 2 \\
& & I & I I & I I I
\end{array} \\
& \begin{array}{lllll}
1 & 1 & 10 & 2 & 2
\end{array} \\
& \text { I II III } \\
& \begin{array}{lllll}
2 & 2 & 1 & 10 & 1
\end{array} \\
& \text { I } I I I \\
& \begin{array}{lllll}
2 & 1 & 2 & 10 & 1 \\
& & & I
\end{array} \\
& \begin{array}{lllll}
2 & 1 & 1 & 10 & 2
\end{array} \\
& \begin{array}{lllll}
2 & 1 & 1 & 10 & 2 \\
& & & I & I I I
\end{array} \\
& \begin{array}{lllll}
1 & 2 & 2 & 10 & 1 \\
& & \text { I } & \text { III }
\end{array} \\
& \begin{array}{lllll}
12 & 1 & 10 & 2
\end{array} \\
& \text { I III } \\
& \begin{array}{ccccc}
1 & 1 & 2 & 10 & 2 \\
& & & & I \\
& I I I
\end{array}
\end{aligned}
$$

In terms of distributions, we have:

$$
\begin{gathered}
\Phi_{1}=\left(\frac{7}{10}, 0,0,0,0\right) \quad \Phi_{2}=\Phi_{3}=\left(\frac{7}{60}, \frac{11}{60}, \frac{1}{5}, 0,0\right) \quad \Phi_{4}=\Phi_{5}=\left(\frac{1}{30}, \frac{1}{10}, \frac{1}{5}, 0,0\right) \\
\Phi_{N C}=\left(0,0,0, \frac{7}{60}, \frac{7}{60}\right) \quad \bar{\Phi}=\left(\frac{42}{137}, \frac{33}{274}, \frac{33}{274}, \frac{9}{137}, \frac{9}{137}\right) \\
B_{1}=\left(\frac{13}{16}, 0,0,0,0\right) \quad B_{2}=B_{3}=\left(\frac{3}{16}, \frac{1}{4}, \frac{1}{16}, 0,0\right) \quad B_{4}=B_{5}=\left(\frac{1}{16}, \frac{1}{8}, \frac{1}{16}, 0,0\right)
\end{gathered}
$$




$$
B_{N C}=\left(0,0,0, \frac{3}{16}, \frac{3}{16}\right) \quad \bar{B}=\left(\frac{195}{548}, \frac{20}{137}, \frac{20}{137}, \frac{35}{548}, \frac{35}{548}\right)
$$

\section{Conclusions}

In this paper, we have introduced a new notion of criticality index that generalizes the classical one of power index to different orders of criticality. It turns out that the representation of the players' interaction in terms of the $n$-dimensional vector containing all criticality indices (where $n$ is the size of the player set) is a good compromise between the exponentially high complexity associated with the characteristic function, and the rough sketch provided by a personal attribution of power to each player. A first attempt to aggregate such criticality indices into a collective one is also presented and studied with respect to several monotonicity properties in weighted majority games. Notice that the majority of the results dealing with the Shapley index and the Banzhaf index in this paper, may be easily adapted to every semivalue Dubey et al. [2081].

An open question for future research concerns a better understanding of the nature of power associated to each index of criticality: how such a power may be carried out by means of the bargaining abilities of players? which trade-off exists between the different orders of criticality and between the corresponding indices?

Another interesting issue is related to an alternative interpretation of the indices of criticality in terms of the likelihood that a coalition will form. For instance, following Example 1, according to the index of criticality of second order, the formation of the grand coalition seems to be more likely in game $u$, where players have a null critical index of second order, than in game $w$, where such an index is one-half for both players. In other words, it is not clear whether the indices of criticality may be used to compare the possibility that a coalition will form over different games with equivalent distribution of (first order) criticality.

\section{Acknowledgments}

The authors gratefully acknowledge two anonymous referees for their useful comments and suggestions that allowed for improving the paper.

\section{References}

Algaba E., Bilbao J.M., van den Brink R., Jiménez-Losada A. [2003] Axiomatizations of the restricted Shapley value for cooperative games on antimatroids. Mathematical Methods of Operations Research 57 : 49-65.

Algaba E., Bilbao J.M., van den Brink R., Jiménez-Losada A. [2004] An axiomatization of the Banzhaf value for cooperative games on antimatroids. Mathematical Methods of Operations Research 59 : 147-166.

Amer R., Carreras F. [2001] Power, cooperation indices and coalition structures. In Holler M.J. and Owen G. (eds.), Power Indices and Coalition Formatio, Dordretch, Kluwer Academic Publishers, pp. 153-173. 
Banzhaf J.F. [1965] Weighted Voting Doesn't Work: A Mathematical Analysis. Rutgers Law Review 19 : 317-343.

Bilbao J.M., Jiménez A., Lopez, J.J. [1998] The Banzhaf power index on convex geometries. Mathematical Social Sciences $36: 157-173$.

Bilbao J.M., Edelman P.H. [2000] The Shapley value on convex geometries. Discrete Applied Mathematics $103: 33-40$.

Brink Van den R. [1997] An axiomatization of the disjunctive permission value for games with a permission structure. International Journal of Game Theory $26: 27-43$.

Brink Van den R., Gilles R.P. [1996] Axiomatizations of the conjunctive permission value for games with permission structures. Games and Economic Behavior 12 : 113-126.

Chessa M., Fragnelli V. [2011] Embedding classical indices in the FP family. AUCO Czech Economic Review 5 : 289-305.

Dall'Aglio M., Fragnelli V., Moretti S. [2016] Orders of Criticality in Voting Games. Operations Research and Decisions $26: 53-67$.

Deegan J., Packel E.W. [1978] A New Index of Power for Simple n-person Games. International Journal of Game Theory $7: 113-123$.

Dubey P. [1975] On the Uniqueness of the Shapley Value. International Journal of Game Theory $4: 131-139$.

Dubey P., Neyman A., Weber R.J. [1981] Value theory without efficiency. Mathematics of Operations Research $6: 122-128$.

Dubey P., Shapley L.S. [1979] Mathematical Properties of the Banzhaf Power Index. Mathematics of Operations Research $4: 99-131$.

Fragnelli V., Ottone S., Sattanino R. [2009] A new family of power indices for voting games. Homo Oeconomicus 26 : 381-394.

Gilles R.P., Owen G., Brink Van den R. [1992] Games with permission structures: the conjunctive approach. International Journal of Game Theory 20 : 277-293.

Holler M.J. [1982] Forming Coalitions and Measuring Voting Power. Political Studies 30 : $262-271$.

Johnston R.J. [1978] On the Measurement of Power: Some Reactions to Laver. Environment and Planning A 10 : 907-914.

Kalai E., Samet D. [1987] On weighted Shapley values. International Journal of Game Theory $16: 205-222$.

Myerson R. [1977] Graphs and Cooperation in Games. Mathematics of Operations Research $2: 225-229$.

Owen G. [1977] Values of Games with a Priori Unions. Lecture Notes in Economic and Mathematical Systems 141 : 76-88.

Shapley L.S., Shubik M. [1954] A Method for Evaluating the Distribution of Power in a Committee System. American Political Science Review 48 : 787-792

Shapley L.S. [1953] A value for n-person games. In: Kuhn H.W. and Tucker A.W. (eds.), Contributions to the theory of games II. Princeton University Press, Princeton, pp. 307-317.

Straffin P.D. [1977] Homogeneity, independence, and power indices. Public Choice 30 : 107-118.

Turnovec F. [1998] Monotonicity and Power Indices. In: Stewart T.J. andvan den Honert R.C. (eds.), Trends in Multicriteria Decision Making, Lecture Notes in Economics and Mathematical Systems 465, Springer-Verlag, pp. 199-214.

Winter E. [1989] A Value for Cooperative Games with Levels Structure of Cooperation. International Journal of Game Theory $18: 227-240$. 